\title{
Neglect und Raumorientierung
}

Michelle Klein, Ann-Kathrin Bur, Georg Kerkhoff

\section{Störungen der visuellen Raumorientierung können sowohl als Symptom innerhalb eines übergeordneten Störungsbildes als auch isoliert auftreten. Darüber hinaus beeinträchtigen das Neglectsyndrom und das Balínt-Holmes-Syndrom die Wahr- nehmung und Orientierung im Raum. In diesem Übersichtsbeitrag werden die drei Störungsbilder vorgestellt.}

\section{Einleitung}

Störungen der visuellen Raumorientierung können sowohl als Symptom innerhalb eines übergeordneten Störungsbildes als auch isoliert auftreten. Meist liegen hierbei distinkte Läsionen unterschiedlicher, überwiegend rechtshemisphärischer parieto-okzipitaler Hirnregionen vor. Symptomatisch äußert sich dies durch Defizite bei der Wahrnehmung von Distanzen, Längen, der Hauptraumachsen und Orientierungen und erschwerten Alltagsleistungen wie Uhrzeit ablesen oder sich Ankleiden.

Es werden 4 verschiedene Formen visueller Raumorientierungsstörungen unterschieden.

- Defizite bei der Wahrnehmung von Distanzen, Längen, der Hauptraumachsen und Orientierungen werden als räumlich-perzeptive Störungen bezeichnet.

- Von räumlich-kognitiven Störungen spricht man, wenn mentale Raumoperationen (Drehung, Spiegelung, Maßstabstransformation) jenseits der Perzeption beeinträchtigt sind.

- Einbußen im manuellen Konstruieren oder Zeichnen einer Gesamtfigur aus einzelnen räumlichen Elementen hingegen definiert man als räumlich-konstruktive Defizite.

- Räumlich-topografische Störungen wiederum zeigen sich in Orientierungsproblemen im realen oder vorgestellten dreidimensionalen, allozentrischen Raum.

Zwei weitere distinkte Störungsbilder, welche die Wahrnehmung und Orientierung eines neurologischen Patienten im Raum beeinträchtigen, sind das Neglectsyndrom und das Balínt-Holmes-Syndrom.

\author{
Man unterscheidet zwischen 4 verschiedenen \\ Formen visueller Raumorientierungsstörungen \\ - räumlich-perzeptive, \\ - räumlich-kognitive \\ - räumlich-konstruktive und \\ - räumlich-topografische.
}

\section{Neglect}

\section{Klinik und Anatomie}

Als Neglect bezeichnet man das Nichtbeachten von sensorischen Reizen (visuell, auditiv, taktil, olfaktorisch) oder in der Vorstellung (repräsentational) sowie die eingeschränkte Benutzung der kontraläsionalen Extremitäten (z. B. vom linken Arm und Bein nach rechtshemisphärischer Hirnschädigung) in der der geschädigten Gehirnhälfte gegenüberliegenden Raumhälfte (hier also der linken). Diese Probleme sind allerdings nicht die Folge elementarer Störungen (wie z. B. einer linksseitigen Hemianopsie oder Hemiparese). Dennoch finden diese Störungen sich oft komorbid. Ein visueller Neglect tritt häufig nach rechtshemisphärischen, etwas seltener auch nach linkshemisphärischen, temporo-parietalen, subkortikalen (Basalganglien, posteriorer Thalamus) oder frontalen Läsionen auf (Übersicht in [1]).

$\begin{array}{ll}\text { ABKÜRZUNGEN } \\ \text { ADL } & \text { Activities of daily Living } \\ \text { BHS } & \text { Balint-Holmes-Syndrom } \\ \text { BORB } & \text { Birmingham Object Recognition Battery } \\ \text { BRS } & \text { Beobachtungsbogen für Räumliche Störungen } \\ \text { CBS } & \text { Catherine-Bergego-Skala } \\ \text { GVS } & \text { galvanisch-vestibuläre Stimulation } \\ \text { OKS } & \text { Optokinetische Stimulation } \\ \text { PA } & \text { visuomotorische Prismenadaptation } \\ \text { tDCS } & \text { transcranial direct current stimulation } \\ \text { TMS } & \text { transkranielle Magnetstimulation } \\ \text { VOSP } & \text { der Visual Object and Space Perception Battery } \\ \text { VS } & \text { Visual Spatial Performance }\end{array}$




\section{FALLBEISPIEL}

\section{Fall 1: Raumorientierung und Neglect links}

Herr M. beklagt das Anstoßen an Hindernissen und Personen, insbesondere in neuer Umgebung. Er habe räumliche Wahrnehmungs- und Orientierungsprobleme, leide unter verschwommenem Sehen, Probleme mit dem Ablesen der Uhrzeit sowie einer Kraftminderung der linken Hand. Auch das Ausführen von zwei Dingen gleichzeitig sei beeinträchtigt, die Aufmerksamkeit eingeschränkt.

Bei Herrn M. handelt es sich um einen 55-jährigen Patienten. 2 Monate zuvor hatte er eine Angiomblutung im parieto-okzipitalen Bereich der rechten Gehirnhälfte erlitten. Die anschließenden bildgebenden Kontrolluntersuchungen bestätigten zwar den Rückgang der Blutung, jedoch wurde zusätzlich ein Infarkt im Bereich des rechten Posteriorstromgebietes und des Balkens sichtbar. Die Anamnese lässt auf räumliche Wahrnehmungsprobleme, einen linksseitigen Neglect sowie einen assoziierten linksseitigen Gesichtsfeldausfall schließen (s.a.

- Abb. 1).
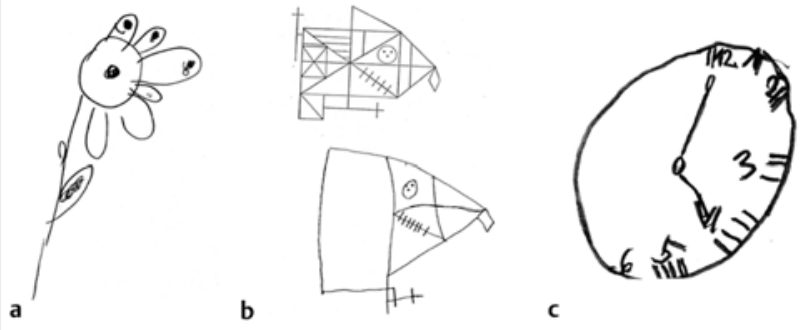

- Abb. 1 Zeichnungen von Herrn M. Zu beachten sind die Auslassungen auf der linken, vernachlässigten Seite in den Zeichnungen.

Patienten zeigen oftmals Probleme beim Essen, Waschen, Rasieren, Schminken, Zeichnen (s.a. > Abb. 1), Rollstuhlfahren, in der Orientierung im Raum und bei zahlreichen anderen Aktivitäten des alltäglichen Lebens. Ursächlich dafür ist die in die ipsiläsionale Raumhälfte (meist rechtsseitig) verschobene visuelle Exploration (Augen- und Kopfbewegungen) [2]. Daraus ergeben sich Folgefehler wie beim Teilen von Objekten zu weit nach ipsiläsional oder die nach ipsiläsional verlagerte subjektive Geradeausrichtung. Auch Kollisionen mit Objekten sowie eine defizitäre Lesefähigkeit (Neglectdyslexie) oder eine Vernachlässigung des eigenen Körpers (Body-Neglect) treten vermehrt im Rahmen einer Neglectproblematik auf. Außerdem können auch Störungen des Lagesinnes (Propriozeption) und der Kinästhetik (Bewegungsvorstellung) an den kontraläsionalen Gliedmaßen bestehen.
Eine wichtige Unterscheidung innerhalb dieses Störungsbildes, neben der Kategorisierung je nach betroffener Sinnesmodalität, ist zudem die Einteilung in raumbezogene versus objektzentrierte Neglectphänomene. Raumbezogene Neglectphänomene beziehen sich auf Raum- oder Körperabschnitte, z. B. übersieht der Patient Objekte auf der kontraläsionalen Seite eines Tisches oder Wörter auf der linken Seite des Textes. Objektzentrierte Neglectphänomene hingegen betreffen die kontraläsionale Seite von Objekten oder Wörtern als Ganzes - unabhängig von der Position des Stimulus im Raum. Beim Lesen können jedoch auch beide Phänomene gemeinsam auftreten. Beispiele sind raumbezogene Auslassungsfehler sowie wort- oder objektbezogene Substitutionsfehler. Der objektzentrierte Neglect tritt im klinischen Setting deutlich seltener auf.

\section{Merke}

Ein essenzieller und nicht zu vernachlässigender Bestandteil des Syndroms ist zudem die fehlende Wahrnehmung der eigenen Krankheit durch die Betroffenen, die sogenannte Unawareness oder Anosognosie.

Die Unawareness oder Anosognosie resultiert aus der Hirnschädigung und ist nicht etwa als Nichtwahrhabenwollen der schweren Beeinträchtigungen zu interpretieren. Dieser Punkt wird in der Differenzialdiagnostik zu Hemianopsiepatienten deutlich: Selbst wenn Betroffene ihre Hemianopsie noch nicht gut kompensieren, beginnen sie nach verbaler Aufforderung, die beeinträchtigte Seite zu explorieren. Beim Vorliegen eines Neglect zeigt sich diese Verhaltensveränderung jedoch nicht oder nur im unmittelbaren zeitlichen Anschluss daran.

\section{FAZIT}

\section{Take Home Message}

Das Nichtbeachten von Reizen verschiedener Sinnesmodalitäten sowie die eingeschränkte Benutzung der kontraläsionalen Extremitäten in der kontraläsionalen Raumhälfte bezeichnet man als Neglect. Die Störung ist meist nach rechtshemisphärischen temporo-parietalen Schädigungen zu finden. Der Neglect kann den ganzen Raum betreffen (raumzentriert), einzelne Objekte (objektzentriert), das Lesen (Neglectdyslexie) oder den eigenen Körper (Body Neglect). Typische Symptome sind das Anstoßen an Hindernissen oder verlängerte Suchzeiten. 


\section{Diagnostik}

Prioritär für die Diagnose des Neglects ist, dass eine primäre sensorische (z. B. Hemianopsie) oder motorische (z. B. Hemiparese) Beeinträchtigung als alleinige Ursache ausgeschlossen werden kann. Diagnostisch bieten sich konventionelle Verfahren wie Durchstreichtests, Linienhalbieren, Lesen und/oder Zeichnen (s.a. - Abb. 1) an. Differenzialdiagnostisch ist der hemianope gegenüber dem Neglect-assoziierten Linienhalbierungsfehler durch die kontra- vs. ipsiläsionale Verschiebung zu unterscheiden (s.a. [3]). Eine systematische Befragung der Angehörigen und/oder Pflegekräfte zu Alltagsbeeinträchtigungen, z. B. durch den Beobachtungsbogen für Räumliche Störungen (BRS), oder die Catherine-Bergego-Skala (CBS), sollte wegen der häufigen komorbid vorliegenden Unawareness der Patienten durchgeführt werden. Auch der Body-Neglect sollte erfasst werden. Eine detaillierte Übersicht der diagnostischen Verfahren findet sich in [1].

Cave

Die Symptomatik ist dem Neglect-Patienten selbst oft nicht bewusst; dieses Phänomen wird als Unawareness oder Anosognosie bezeichnet. Eine reine Befragung des Betroffenen reicht bei bestehendem Verdacht also nicht aus. Das Verhalten in Alltagssituationen, während der Therapie oder die Befragung der Angehörigen kann wichtige Informationen liefern.

\section{Spontanverlauf, Prognose und Therapie}

Viele Patienten (30-40\%) zeigen auch nach einem Jahr noch deutliche Einbußen. Patienten zeigen eine bevorzugte Hinwendung zur ipsiläsionalen Seite in Alltagssituationen sowie beim Absuchen komplexer Suchvorlagen sowie eine gestörte Verarbeitung von zwei bilateral (links und rechts simultan) dargebotenen Reizen (Beispiel: Auto links, Person rechts, Patient berichtet nur den Reiz rechts). Dieses Phänomen bezeichnet man als Extinktion (von engl. „to extinguish“=löschen).

Die spezifische Neglecttherapie richtet sich nach den konkreten Beeinträchtigungen und dem Akutheitsgrad des Patienten. Ziel aller therapeutischen Bemühungen sollte die Verbesserung der Alltagsleistungen des Patienten sein, damit dieser zunehmend selbstständiger in seinen Aktivitäten werden und in Alltag und Beruf teilhaben kann. Zudem sollten einzelne Ansätze miteinander kombiniert werden. Die folgende Infobox fasst die wichtigsten Therapieverfahren und -techniken für die Neglectbehandlung zusammen (mehr Details in [1].
THERAPIE

Therapieverfahren: Neglect

Neuromodulation

Repetitive „Brain Stimulation“ des (ungeschädigten) parietalen Kortex (durch TMS, tDCS) reduziert die Hemmung der geschädigten Hemisphäre durch parietale Regionen der ungeschädigten Hemisphäre und vermindert so den visuellen $\mathrm{Ne}$ glect und Alltagsdefizite (ADL) dauerhaft.

Optokinetische Stimulation mit Blickfolgebewegungen Verbesserung multimodaler Neglectdefizite durch die Aktivierung des visuellen und teilweise des vestibulären Kortex, aber auch parieto-temporaler Hirnregionen beider Hemisphären. Signifikante und dauerhafte Verbesserung des visuellen und akustischen Neglects nach 5-20 Therapiesitzungen. Die Durchführung von Blickfolgebewegungen durch den Patienten ist wichtig für die Wirksamkeit des Verfahrens. „Passive“ Stimulation ist wirkungslos.

\section{Galvanisch-vestibuläre Stimulation (GVS)}

GVS aktiviert das thalamokortikale vestibuläre System und verbessert das „Körper-im-Raum-Empfinden“ bei Neglect und Extinktion. GVS verbessert den Lagesinn und reduziert taktile Extinktion dauerhaft. GVS verbessert auch die visuelle und haptische subjektive Vertikaleneinschätzung - verbessert also räumlich-perzeptive Leistungen ebenfalls.

\section{Aufmerksamkeitstraining}

Die Verwendung von Alertnessreizen führt zur besseren Ausrichtung der Aufmerksamkeit in den vernachlässigten Halbraum. Eine Steigerung der Daueraufmerksamkeit reduziert die nicht-lateralisierten Aufmerksamkeitsdefizite bei Neglectpatienten. Durch das Training kommt es zu fronto-parietalen Mehraktivierungen bei Neglectpatienten.

\section{Visuelles Explorationstraining}

Verbesserung der Suchstrategien (systematischer) und dadurch Reduktion der Auslassungen in der visuellen Suche. Auf diese Weise werden Verbesserungen der visuellen Exploration, des Lesens und ein partieller Transfer auf Alltagsleistungen erreicht. Nackenmuskelvibration

Die Vibration der kontraläsionalen Nackenmuskeln aktiviert das propriozeptive System, die Inselregion und den superioren temporalen Kortex. Die Nackenmuskelvibration verbessert Explorationsleistungen (visuell und taktil) und das subjektive Geradeausempfinden des Patienten im Raum.

\section{Visuomotorische Prismenadaptation (PA)}

Ausnutzen des sensomotorischen Rekalibrierungseffekts nach Tragen (15 min) eines Prismas (Blickverlagerung um 10-15 zur ipsiläsionalen Seite). Es kommt vermutlich zu einer Rekalibrierung der gestörten Raumorientierung durch die Verbesserung von Aufmerksamkeits- und Explorationsleistungen. PA aktiviert zerebelläre und parietale Hirnregionen bei Neglectpatienten.

\section{Periphere Magnetstimulation}

Die magnetische Stimulation der Hand ist schmerzfrei und aktiviert den kontralateralen, somatosensorischen Kortex. Dies führt zu einer Aktivierung der geschädigten Hemisphäre und reduziert die taktile Extinktion und den körperbezogenen $\mathrm{Ne}$ glect. 
Die beschriebenen Therapieverfahren sind zu unterschiedlichen Zeitpunkten im Rehabilitationsverlauf sinnvoll:

In der Akutphase (0-4 Monate nach der Erkrankung) geht es um eine möglichst rasche und intensive Stimulation des Patienten auf allen möglichen und für ihn angenehmen sensorischen Kanälen mit dem Ziel, die multimodale Vernachlässigungssymptomatik zu vermindern. Hier bietet sich vor allem die optokinetische Stimulationstherapie an, bei der der Patient bewegte Punktemuster am Bildschirm mit den Augen aktiv verfolgt, um den vernachlässigten Halbraum besser zu explorieren.

Zum Ende dieser Aktivationsphase sollten dann vermehrt strategiebezogene Kompensationsverfahren eingesetzt werden, die wichtige Alltagsfertigkeiten wie etwa koordinierte Augen- und Kopfbewegungen (Blickstrategie) zur vernachlässigten Seite vermitteln.

Zum Ende dieser Stabilisationsphase sollten dann alltagsnähere Therapieverfahren eingeführt werden, die das direkte Üben wichtiger Alltagshandlungen zunehmend unter realen Alltagsbedingungen mit interferierenden Störreizen, Zeitdruck und komplexerem Anforderungsprofil üben (z. B. die Teilung der Aufmerksamkeit beim gleichzeitigen Gehen und Explorieren des Raumes durch den Patienten). In dieser Phase sollten Strategien aus der vorhergehenden Kompensationsphase in konkrete Alltagsabläufe eingebunden werden (Beispiel: ein Obstregal mit Augenbewegungen absuchen; gleichzeitig stehen oder gehen und explorieren; sich unterhalten und gehen).

Auch nach Entlassung des Neglectpatienten aus der Rehabilitationsklinik sollte weiterhin ambulant oder teilstationär Neglecttherapie angeboten werden.

\section{FAZIT}

\section{Take Home Message}

Verfügbare Therapieverfahren sollten massiert eingesetzt werden, da sie oftmals erst nach mehreren Therapiesitzungen einen Effekt zeigen. Neben klassischen Stimulationsansätzen bieten sich neuerdings auch Verfahren der Neuromodulation an (s. o., Infobox).

Strategieorientierte Verfahren sind vor allem für den Alltagstransfer wichtig. Eine Kombination aus mehreren Therapieformen verspricht größeren Erfolg, sollte aber nicht exakt gleichzeitig, sondern leicht zeitlich versetzt erfolgen. Zudem empfiehlt sich eine an den Reha-Aufenthalt anschließende ambulante oder teilstationäre Fortsetzung der neuropsychologischen Therapie.

\section{Räumliche Orientierungsstörungen}

Es werden vier verschiedene Formen visueller Raumorientierungsstörungen unterschieden: räumlich-perzeptive, räumlich-kognitive, räumlich-konstruktive und räumlich-topografische. Diese 4 Störungsformen werden im Folgenden separat hinsichtlich ihrer klinischen und anatomischen Besonderheiten erläutert.

\section{Klinik und Anatomie räumlich-perzeptiver Störungen}

Unter räumlich-perzeptiven Störungen subsumiert man unterschiedliche Defizite bei elementaren perzeptiven Leistungen (s. $>$ Abb. 2).

\section{Störungen der subjektiven Hauptraumachsen (subjektive visuelle Vertikale und Horizontale)}

Sie zeigen sich in Abweichungen von der tatsächlichen Vertikalen bzw. Horizontalen bei der Beurteilung, wann eine Linie, die schrittweise rotiert wird, vertikal bzw. horizontal ausgerichtet ist. Es treten nicht nur Verdrehungen der Vertikalen bzw. Horizontalen auf, sondern häufig kommt es zu Verdrehungen des gesamten visuellen und taktilen Raums in der Frontalebene [4]. Darüber hinaus wurden auch Abweichungen der subjektiven Vertikalen in der Sagittalebene beobachtet [5], d.h. wenn beurteilt werden soll, wann ein Stab, der vor und zurück rotiert werden kann, vertikal ausgerichtet ist. Ein Neglect und/oder eine Hemianopsie aggravieren die Abweichung der subjektiven Vertikalen. Dieses Verhalten ist auch Teil der Problematik des Pusher-Syndroms.

Störungen der subjektiven Hauptraumachsen treten häufig nach rechtsseitiger, temporo-parietaler, seltener nach entsprechender linksseitiger Hirnläsion auf. Sie finden sich ebenfalls nach Schädigungen des Thalamus (Nucleus ventro-posterolateralis), des Hirnstamms, inferiorer okzipito-frontaler und superior-longitudinaler Faserverbindungen sowie peripher-vestibulärer Areale. Bei Patienten mit linksseitigen Schädigungen mit Störungen der subjektiven Vertikalen scheint vor allem der insuläre Kortex, und bei rechtsseitigen Schädigungen der inferiore Gyrus frontalis und der superiore Gyrus temporalis betroffen zu sein. Es überwiegen kontraversive Abweichungen der Vertikalen, v.a. nach rechtshemisphärischer Läsion [6]. 
Probleme bei der Orientierungsschätzung (Neigungswinkel von Reizen)

Als Orientierungsschätzung bezeichnet man die Fähigkeit zur Unterscheidung verschiedener Neigungswinkel von Linien oder Objekten (nicht zu verwechseln mit räumlich-topografischen Orientierungsstörungen). Zu Verwechslung ähnlicher Orientierungen (z.B. $30^{\circ}$ versus $40^{\circ}$ ) kommt es vor allem bei kurzen Linien. Personen mit einer entsprechenden Hirnläsion haben diese Fähigkeit zur Unterscheidung der Neigungswinkel oft verloren, ohne sich dessen selbst bewusst zu sein. Hierbei verursachen rechtshemisphärische Läsionen häufigere und schwerere Defizite der visuellen Orientierungsschätzung und der subjektiven Hauptraumachsen als linkshemisphärische Läsionen. Die visuelle Horizontale und die Einschätzung schräger Orientierungen sind normalerweise in einem ähnlichen Ausmaß betroffen wie die Rotationen der visuellen Vertikalen. Der ganze Raum ist dadurch „verkippt“ - dies ist den Patienten aber nur selten subjektiv bewusst. Nach linkshemisphärischer, supratentorieller Läsion kommt es überwiegend zur Rotation des Koordinatensystems im Uhrzeigersinn, nach rechtshemisphärischer Läsion meist gegen den Uhrzeigersinn.

Entsprechende Defizite finden sich häufig nach rechts temporo-parietaler Schädigung sowie nach Stammganglienläsion rechts, selten auch nach linksfrontalen Läsionen [7]. Rechtshemisphärische Läsionen verursachen häufigere und schwerere Defizite der Orientierungsschätzung. Hierbei können die Defizite je nach Größe der Läsion sich sehr spezifisch äußern, da viele visuelle Neurone Orientierungsspezifität aufweisen. Bildgebenden Studien zufolge sind der obere Scheitellappen, der laterale okzipitale Kortex und prämotorische Areale an der visuellen Orientierungsschätzung beteiligt [8], mit rechtshemisphärischer Akzentuierung.

- Abb. 2 veranschaulicht einige räumliche Tests exemplarisch, die im Programm Visual Spatial Performance (VS) [9] verwendet werden.

\section{Defizite in der Längenschätzung (Ausdehnung} innerhalb von Objekten)

Die Längenschätzung spezifiziert den horizontalen oder vertikalen Raum innerhalb eines Objektes oder einer Fläche und definiert die Fähigkeit einer Person, die Länge von Objekten einschätzen zu können. Störungen der Längenschätzung finden sich oft in Kombination mit Neglect. Dementsprechend finden sich Störungen oft nach okzipito-parietalen Läsionen.

\section{Defizite in der Distanzschätzung (Ausdehnung zwischen Objekten)}

Im Gegensatz dazu geht es bei der Distanzschätzung um räumliche Abstände zwischen Objekten. Störungen der Längen- und Distanzschätzung treten häufig komorbid

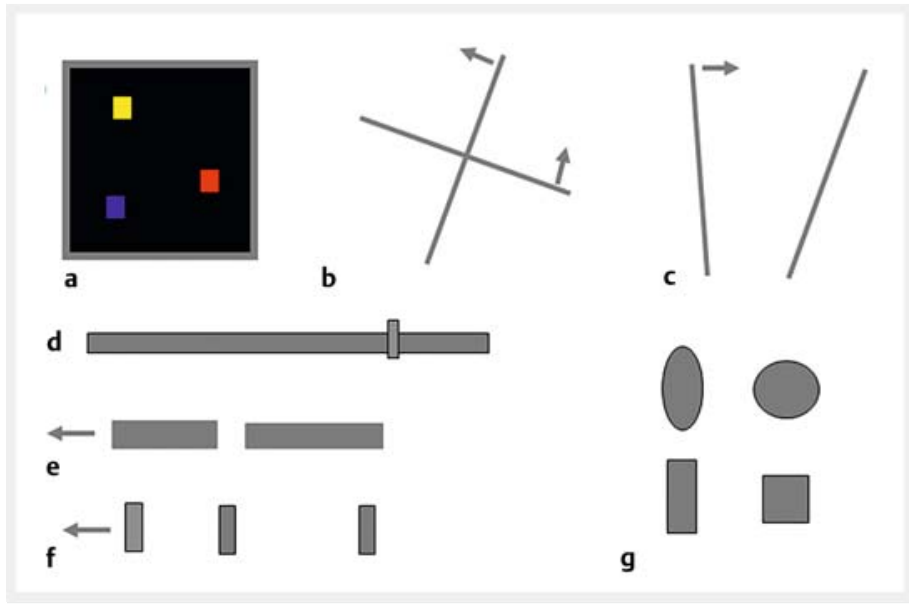

- Abb. 2 Veranschaulichung räumlicher Testverfahren nach Visual Spatial Performance (VS) [9]. a Positionswahrnehmung: der Patient muss die gezeigte Vorlage auf ein neues Quadrat richtig übertragen. b Subjektive Achse: der Patient bringt das Kreuz in eine für ihn aufrechte Position. c Orientierungsschätzung: der Patient soll die Neigung der linken Linie an die der rechten Linie anpassen. $\mathbf{d}$ Linienhalbierung: der Patient kann den Regler bewegen und damit die Linie subjektiv halbieren. e Längeneinschätzung: der Patient soll das kürzere oder längere (je nach Frage) Rechteck identifizieren. f Distanzschätzung: der Patient soll das nähere oder entferntere Rechteck in Relation zum mittigen Rechteck identifizieren (je nach Frage). g Formwahrnehmung: der Patient soll eine Form identifizieren nach der gefragt ist.

auf, können in Einzelfällen aber auch unabhängig voneinander vorliegen. Patienten mit entsprechenden Defiziten schätzen die „Geometrie“ des Raumes als zu klein oder zu groß ein. Ausgeprägte Defizite in der visuellen Distanz- und Entfernungsschätzung zeigen Patienten mit bilateralen parieto-okzipitalen Hirnläsionen, beispielsweise beim Balínt-Holmes-Syndrom (s.u.).

Die zu beobachtenden Veränderungen in der wahrgenommenen Ausdehnung des Raumes stehen vermutlich in Zusammenhang mit den entsprechenden Störungen im subjektiven Geradeausempfinden (s.u.) sowie Mechanismen der Blicksteuerung und der visuellen Raumexploration (visuelles Abtasten der Positionen im Raum, für die ein Distanzvergleich vorgenommen werden soll).

Abweichungen in der subjektiven Geradeausrichtung und bei der Einschätzung der subjektiven Mitte in der Linienhalbierung

Abweichungen in der Linienhalbierung und/oder subjektiven Geradeausrichtung finden sich sowohl bei Patienten mit Neglect als auch bei Patienten mit homonymen Gesichtsfeldausfällen. Ihnen ist es im Alltag beispielsweise nicht mehr möglich, Gegenstände in der Mitte zu halbieren, eine Strecke zu Fuß im Flur zu gehen oder Objekte auf einem Tisch zu positionieren. Die Verschiebung der subjektiven Mitteneinschätzung erfolgt bei den Hemianopsiepatienten ohne Neglect 
nach kontraläsional, bei den Neglectpatienten in der Frühphase nach ipsiläsional. Treten beide Störungen gemeinsam auf (Hemianopsie plus Neglect), wirkt sich der Neglect deutlicher auf die Halbierung aus als die Hemianopsie. Ipsiläsionale Verschiebungen der Geradeausrichtung treten nach Läsionen perisylvischer Regionen auf. Neuere Erkenntnisse weisen allerdings darauf hin, dass sich diese Unterscheidung nicht in der akuten Phase nach der Schädigung zeigt [3].

\section{Defizitäre Positionswahrnehmung (relativ, absolut)}

Die gestörte Positionsschätzung verursacht einen Genauigkeitsverlust (größere Variabilität) sowie eine systematische Verschiebung der reproduzierten Positionen zu einer Seite. Letzteres steht oft im Zusammenhang mit der Verschiebung der subjektiven Geradeausrichtung bei Neglect oder Hemianopsie. Man unterscheidet die relative und die absolute Positionsschätzung. Defizite in der relativen Positionsschätzung zeigen sich bei der fehlerhaften Kodierung der Raumposition eines Reizes bei gleichzeitig vorhandenem Referenzsystem, wodurch es einem Patienten z.B. nicht möglich ist, ein Kreuz auf einem Blatt Papier auf ein anderes Blatt zu kopieren. Absolute Positionsschätzung dagegen bezeichnet die Fähigkeit, die Position eines Reizes ohne räumliche Referenz zu kodieren (z.B. im Perimeter oder im Dunkeln).

Defizite der relativen und absoluten Positionsschätzung treten häufig, jedoch nicht ausschließlich nach rechtshemisphärischer Hirnschädigung auf, insbesondere bei Patienten mit superior-parietalen Läsionen. Bei der absoluten Positionsschätzung zeigen auch Patienten mit parietalen Läsionen eine ungenauere Lokalisation kurzzeitig im kontralateralen Gesichtsfeld dargebotener Reize.

\section{FAZIT}

\section{Take Home Message}

Räumlich-perzeptive Störungen betreffen elementare Sehleistungen. Hierzu gehören Defizite der subjektiven Hauptraumachsen, der Orientierungsschätzung, der Längenschätzung, der Distanzschätzung, der Geradeausrichtung und der Linienhalbierung sowie eine gestörte Positionswahrnehmung. Diese Defizite führen in Kombination zu einer fehlerhaften Eigenbewegung im Raum und zu einer gestörten Interaktion mit externen Objekten. Kritische Regionen für das Auftreten eines solchen Symptomkomplexes sind primär rechtshemisphärisch im parieto-okzipito-temporalen Bereich zu finden.

\section{Klinik und Anatomie räumlich-kognitiver Störungen}

Von räumlich-perzeptiven Defiziten sind räumlich-kognitive Störungen (oder Einbußen visueller Raumoperationen) zu unterscheiden, die über die Wahrnehmungsleistung hinaus, oder ohne eine solche, eine mentale Raumoperation erfordern (z. B. mentale Rotation, Maßstabstransformation, Spiegelung). Neben parietalen Regionen sind für die mentale Rotation auch die frontalen Augenfelder, der obere Scheitellappen und Areale des mittleren Temporallappens wichtig $\left(V_{5}\right)$. Für räumliche Transformationen anderer Art (Spiegelung) ist der parieto-okzipitale Kortex bedeutsam.

\section{Klinik und Anatomie räumlich-konstruktiver Störungen}

Räumlich-konstruktive Störungen wiederum zeichnen sich durch die Unfähigkeit aus, einzelne Elemente einer Figur mit der Hand zu einem Ganzen zusammenzusetzen (Zeichnen, Würfel zusammenfügen). Die damit verbundenen visuomanuellen Fertigkeiten sind Bestandteil vieler komplexer Handlungsabläufe im Alltag, sodass Störungen dieser Fähigkeit Probleme und Einschränkungen im Alltag zur Folge haben (Ankleiden, Transfers, Paketpacken, Rollstuhlnavigation).

Dabei sollten zum Problemkomplexe betrachtet werden: Zum einen räumlich-perzeptive Defizite [10] und zum anderen eine gestörte Erinnerung („Remapping“) der zuvor fixierten Positionen auf der Vorlage, wenn eine Person einen Blickwechsel zwischen einer Vorlage und seiner Kopie durchführt [11]. Die Ortsinformationen der zuvor betrachteten Elemente der Vorlage scheinen hierbei verloren zu gehen, was zur Folge hat, dass die Kopie an der falschen Position angefertigt wird bzw. erscheint und somit von der Vorlage abweicht.

Räumlich-konstruktive Defizite treten nach fokalen parietalen, frontalen und subkortikalen sowie diffus-disseminierte Schädigungen auf [12]. 


\section{Klinik und Anatomie räumlich-topografischer Störungen}

Von den räumlich-konstruktiven Störungen wiederum abzugrenzen sind räumlich-topografische Störungen. Diese zeichnen sich durch Navigationsdefizite im vorgestellten oder realen dreidimensionalen Raum aus. Wichtige Landmarken können von Betroffenen nicht mehr erkannt werden, und so verirren diese sich sowohl in vertrauten als auch in neuen Umgebungen, vor allem bei Dämmerung oder bei Betrachtung aus einer anderen Perspektive. So können sich Patienten keine räumliche Vorstellung über Orte in ihrer Umgebung erstellen und haben folglich Probleme beim Wegelernen. Räumlich-topografische Probleme treten auch bei Patienten mit Neglect auf, sind hier jedoch als Folge der Vernachlässigung zu werten.

Schwerste topografische Orientierungsstörungen kommen darüber hinaus nach bilateralen Läsionen beim Balínt-Syndrom vor. Hier sind die Fixationsstörung, die Simultanagnosie und die gravierenden räumlichen Störungen ursächlich. Humanstudien mit bildgebenden Verfahren und an Patienten belegen die Bedeutung hippocampaler, parahippocampaler und retrosplenialer Regionen für die räumliche Navigation [13]. Dementsprechend sind räumlich-topografische Störungen nach Läsionen des ventralen visuellen Pfades zu erwarten (parahippocampale Läsionen). Als sekundäres Defizit treten sie wie bereits erwähnt auch im Gefolge anderer neuropsychologischer Störungen auf.

\section{Anatomie}

Räumlich-kognitive Störungen betreffen Leistungen, die über die rein perzeptuelle Leistung hinausgehen. Hierbei geht es um die kognitiven Operationen mit dem perzeptuellen Input. Räumlich-konstruktive Störungen stellen ein eher praktisches Defizit dar, bei denen Patienten Probleme mit den visuomanuellen Fertigkeiten haben. Dies erschwert die Interaktion mit externen Objekten. Räumlich-topografische Störungen führen zu Navigationsdefiziten in der Vorstellung als auch in der realen Umsetzung der Patienten. Hierbei haben die Patienten oft Schwierigkeiten, sich in bekannten und mehr noch in neuen Umgebungen zurechtzufinden. Verglichen mit räumlich-perzeptiven Störungen sind die Läsionsorte variabler und umfassen auch frontale sowie subkortikale Regionen.

\section{Diagnostik}

- Tab. 1 gibt einen Überblick über die wichtigsten Diagnostikverfahren für die unterschiedlichen räumlichen Störungen (Details in [4]).
FALLBEISPIEL

\section{Fall 2: Räumlich-topografische-Störungen}

Frau S. berichtet im Anamnesegespräch Probleme, sich allein in ihrem Zuhause und ihrer gewohnten Umgebung zurechtzufinden. Sie sei früher jeden zweiten Morgen eine bestimmte Strecke entlang spazieren gegangen. Doch als sie nach dem Schlaganfall das erste Mal versucht habe, den Weg zu finden, habe sie sich verlaufen. Auch nachdem sich ihr Zustand verbessert habe, wäre es ihr nicht möglich gewesen, allein den Weg zu finden. Sie sei zunehmend verunsichert, welche Richtung sie, ausgehend von ihrem Haus, einschlagen solle und an welchem Haus sie Richtung Wald abbiegen müsse. Ihr Mann sei die Strecke schon öfter mit ihr gemeinsam gegangen. Dabei habe sie versucht, sich den Weg genauestens einzuprägen. Jedoch würde sie immer wieder scheitern, beim Versuch den Weg allein zurückzulegen. Zudem begäbe sie sich in ihrem Haus oftmals auf die Suche nach dem Badezimmer (welches sich neben dem Wohnzimmer befindet). Sie lande dann öfter mal in der Küche oder im Arbeitszimmer. Es fiele ihr zunehmend schwer, sich den Plan ihres eigenen Zuhauses zusammenhängend vorzustellen.

Die Patientin erlitt im Jahr vor der Untersuchung einen Schlaganfall der rechten A. cerebri media mit Schädigungen im medialen bis inferior-posterioren Temporallappen. Ein generelles Gedächtnisdefizit wurde nicht berichtet, der Hippocampus blieb laut Bildgebung unbeschädigt.

\section{Merke}

Für die Diagnostik von visuellen Raumorientierungsstörungen können sowohl computerbasierte als auch Paper-and-Pencil-Verfahren herangezogen werden. Je nach Indikation sollten unterschiedliche Tests herangezogen werden, um eine differenzierte Diagnose zu erhalten.

\section{Spontanverlauf, Prognose und Therapie}

Nach einer teilweisen Rückbildung visuell-räumlicher Orientierungsstörungen in den ersten 3 Monaten nach der Schädigung zeigen noch ca. 30 \% der Patienten behandlungsrelevante Defizite. Die Behandlung der Störungen ist mitunter entscheidend für einen günstigen Rehabilitationsverlauf. Tab.2 gibt einen Überblick über die wichtigsten Verfahren (nach [4]).

\section{Merke}

Nicht alle in der Therapie gemachten Fortschritte übertragen sich auf den Alltag der Patienten. Deshalb ist es wichtig, relevante Funktionen in die Therapie einzubauen und konkret einzelne Leistungen zu trainieren. Dies erfordert eine detaillierte Analyse der Anforderungen, mit denen der Patient sich in seinem täglichen Leben konfrontiert sieht, sobald er die das stationäre Setting verlässt. 
- Tab. 1 Diagnostische Verfahren zur Erfassung räumlich-perzeptiver (RP), räumlich-kognitiver (RO) und räumlich-konstruktiver Störungen (RK). Die Abkürzungen geben an, für welchen Bereich räumlicher Störungen sich welche Verfahren diagnostisch eignen.

\begin{tabular}{|c|c|c|c|}
\hline Typ & Testverfahren & Diagnostisches Prinzip & Bewertung \\
\hline RP & $\begin{array}{l}\text { Judgement of Line Orientation } \\
\text { (Benton et al. 1983) [14] }\end{array}$ & Prüfung der Linienorientierung & leicht durchführbar; 2 Paralleltests; auch für Kinder \\
\hline RP, RO & $\begin{array}{l}\text { Visual Spatial Performance (VS) } \\
\text { (Kerkhoff u. Marquardt 2004) [9] }\end{array}$ & $\begin{array}{l}\text { PC-Verfahren zur Analyse } 10 \text { räumlich- } \\
\text { perzeptiver Leistungen und räumlicher } \\
\text { Gedächtnisleistungen } \\
\text { räumlich-kognitive Tests } \\
\text { Optokinetik } \\
\text { Feedback-Therapie }\end{array}$ & $\begin{array}{l}6 \text { normierte klinische Standarduntersuchungen } \\
\text { sowie zahlreiche Tests mit Cut-off-Werten; keine } \\
\text { Wiederholungseffekte; geeignet für Verlaufsmes- } \\
\text { sungen und Feedback-Therapie }\end{array}$ \\
\hline $\mathrm{RP}, \mathrm{RO}$ & $\begin{array}{l}\text { Visual Object and Space Percep- } \\
\text { tion Battery (VOSP) (Warrington } \\
\text { u. James 1992) [15] }\end{array}$ & $\begin{array}{l}4 \text { räumliche Subtests: Positionsvergleich, } \\
\text { Zahlenlokalisieren, Punktezählen, Würfel- } \\
\text { zählen }\end{array}$ & $\begin{array}{l}\text { normierter Test; der Untertest Punktezählen ist eine } \\
\text { visuelle Explorationsaufgabe; keine Paralleltests }\end{array}$ \\
\hline $\mathrm{RP}, \mathrm{RO}$ & $\begin{array}{l}\text { Birmingham Object Recognition } \\
\text { Battery (BORB) (Riddoch u. } \\
\text { Humphrey 1993) [16] }\end{array}$ & $\begin{array}{l}4 \text { räumliche Subtests: Linienorientierung, } \\
\text { Würfelzählen, Längen-, Größen- und Posi- } \\
\text { tionsschätzung innerhalb eines Objekts }\end{array}$ & $\begin{array}{l}\text { normierter Test mit theoretischer Einbettung; zur } \\
\text { Untersuchung von } 4 \text { räumlich-perzeptiven Leistun- } \\
\text { gen geeignet }\end{array}$ \\
\hline RO, RK & $\begin{array}{l}\text { räumliche Subtests aus Intelli- } \\
\text { genztests }\end{array}$ & Subtests aus IST, LPS, HAWIE (K) & Subtests aus IST, LPS, HAWIE (K) \\
\hline RO, RK & Mosaiktest & $\begin{array}{l}\text { Konstruktion dreidimensionaler Muster } \\
\text { nach Vorlage }\end{array}$ & $\begin{array}{l}\text { nicht für Verlaufsmessung geeignet; zeitgebunde- } \\
\text { ner Test }\end{array}$ \\
\hline RO, RK & Zeichenaufgaben (2D, 3D) & Zeichnen von Haus, Blume, Uhr & $\begin{array}{l}\text { leicht durchführbar (am Krankenbett möglich); } \\
\text { hohe Augenschein-Validität; nicht normiert }\end{array}$ \\
\hline $\begin{array}{l}\text { AbkürzL } \\
\text { HAWIE }( \\
\text { IST }=\text { Inte } \\
\text { LPS = Lei } \\
\text { RK= räur } \\
\text { RO= räu } \\
\text { RP = räur }\end{array}$ & $\begin{array}{l}\text { en } \\
\text { Hamburg-Wechsler-Intelligenztest für } \\
\text { enz-Struktur-Test } \\
\text { רgsprüfsystem } \\
\text { h-konstruktive Störung } \\
\text { ch-kognitive Störung } \\
\text { h-perzeptive Störung }\end{array}$ & vachsene (Kinder) & \\
\hline
\end{tabular}

\ab.2 Schematische Übersicht über Therapieansätze bei räumlichen Störungen.

\begin{tabular}{|l|l|l|}
\hline Ansatz & Therapeutisches Prinzip & Bewertung \\
\hline $\begin{array}{l}\text { Feedbackbasiertes Training räumlich- } \\
\text { perzeptiver Leistungen (Funk et al. } \\
\text { 2012) [17] }\end{array}$ & $\begin{array}{l}\text { Neukalibrierung der räumlichen Wahrnehmung durch ab- } \\
\text { gestuftes Training mit visuellem Feedback }\end{array}$ & $\begin{array}{l}\text { Transfer auf untrainierte Leistungen und } \\
\text { den Alltag }\end{array}$ \\
\hline $\begin{array}{l}\text { Optokinetische Stimulation (OKS) } \\
\text { Darbietung spezifischer visueller } \\
\text { Hintergrundbewegung) (Kerkhoff } \\
\text { 2003) [18] }\end{array}$ & $\begin{array}{l}\text { Ausnutzung des aufmerksamkeitsfördernden Effektes von } \\
\text { OKS für Distanz- und Längenschätzung sowie Hauptraum- } \\
\text { achsen durch wiederholte Stimulation }\end{array}$ & $\begin{array}{l}\text { besonders geeignet bei assoziiertem } \\
\text { Neglect und geringer Awareness; wirkt } \\
\text { vermutlich multimodal }\end{array}$ \\
\hline $\begin{array}{l}\text { Räumlich-konstruktives Training } \\
\text { (Weinberg et al. 1982) [19] }\end{array}$ & $\begin{array}{l}\text { Verbesserung räumlich-perzeptiver, -konstruktiver und } \\
\text { planerischer Leistungen durch Tangram-, Valenser- und } \\
\text { Mosaiktesttraining }\end{array}$ & positive Effekte auf Selbsthilfeleistungen \\
\hline $\begin{array}{l}\text { Alltagsorientierte Therapie } \\
\text { Üben „räumlicher“ Alltagshandlungen: z. B. Rollstuhlfahren, } \\
\text { Ankleiden, Wäsche zusammenlegen }\end{array}$ & $\begin{array}{l}\text { sinnvoll zur Verbesserung der Alltagsleis- } \\
\text { tungen und Awareness }\end{array}$ \\
\hline
\end{tabular}




\section{Balínt-Holmes-Syndrom}

Patienten mit einem Balínt-Holmes-Syndrom zeigen im Alltag oftmals Probleme beim Greifen nach Gegenständen (die nicht Folge einer Lähmung sind), sie kollidieren mit Hindernissen, haben einen eingeschränkten Überblick und Schwierigkeiten, Distanzen und Positionen richtig einzuschätzen. Die elementaren visuellen Leistungen der Patienten sind oftmals intakt. Oft werden solche Patienten initial fälschlich für „blind“ gehalten, können aber einzelne Details (z.B. eine Fluse auf dem Arztkittel) erstaunlich gut wahrnehmen.

\section{Klinik und Anatomie}

Das Balínt-Holmes-Syndrom (BHS) umschreibt einen Symptomkomplex aus

- Simultanagnosie,

- optischer Ataxie,

- Raumverarbeitungsstörungen und

- Blickbewegungsstörungen.

Die Symptome können sowohl im Rahmen des Vollbilds des BHS auftreten als auch isoliert. $>$ Tab. 3 illustriert die wichtigsten Fakten zum BHS.

\section{Diagnostik}

Bei Verdacht auf BHS ist ein detailliertes Assessment der konstituierenden Symptome erforderlich. Es ist anzunehmen, dass die Erkrankung oft übersehen oder fehldiagnostiziert wird (z.B. als Röhrengesichtsfeld oder zerebrale Blindheit).

\section{Spontanverlauf, Prognose und Therapie}

Über die Rückbildung und Behandlung solcher Störungen ist wenig bekannt. Trotz der meist bleibenden Grundstörung kann ein alltagsorientiertes Training zu Verbesserungen führen [20]. Systematische Behandlungsansätze existieren bislang kaum. Es sollte gezielt nach (teilweise) erhaltenen Leistungen gesucht werden (z.B. gute Gedächtnisleistungen, intakte Augenfolgebewegungen, intakte Lernleistungen, erhaltene Farb- und Bewegungswahrnehmung), die zur Kompensation eingesetzt werden können. Folgende Anregungen können für die Behandlung gegeben werden.

\section{Fixation verbessern}

In der Behandlung sollte zunächst versucht werden, die visuelle Aufmerksamkeit des Patienten durch Verwendung gut sichtbarer, farbiger Alltagsobjekte zu gewinnen (etwa ein Farbstift, Tennisball). Patienten mit BHS reagieren eher auf bewegte als auf statische Reize (optokinetische Muster lösen oft Folgebewegungen aus), da bewegte Reize eher bemerkt werden. Offensichtlich ist es mit Hilfe solch bewegter Reize eher möglich, die spasmodische Fixation zu lösen und wieder an ein neues Objekt zu binden. Selbst schwer gestörte Patienten mit Balínt-Holmes-Syndrom können meist noch in be-

\section{FALLBEISPIEL}

\section{Fall 3: Balínt-Holmes-Syndrom}

Es handelt sich um den Dialog zwischen einer Patientin mit Balínt-Syndrom 3 Monate nach bilateralen Posterior-mediaGrenzzoneninfarkten mit beidseitigen parieto-okzipitalen Läsionen $(\mathrm{P})$ und ihrem Untersucher $(\mathrm{U})$.

U: „Wie empfinden Sie Ihr Sehen seit den Schlaganfällen?“ P: „Von Anfang an war es so, dass ich ganz erstaunlich kleine Dinge oft ganz scharf gesehen habe. Auch Details habe ich ganz klar gesehen. Am dritten Tag, da hat mich mal ein Nachtpfleger in der Früh aufgeweckt und mir gesagt, ich zeige Ihnen jetzt den Sonnenaufgang, und es war ein völlig normales Bild. Aber es war so ein gesplittertes Bild, wie ... durch ein Kristall ... so ein gebrochenes Bild ... wie ein gebrochenes Glas und parallel verschoben ...“

U: „Wie war denn das mit dem Greifen, war das schwierig?“

P: „Ja, total. Das ist es ja immer noch. Manchmal denke ich, es ist etwas direkt da und ich brauche bloß hingreifen und dann ist nichts da ... oder ich greife eben zu nahe."

U: „Sehen Sie die Tiefe bei Treppenstufen?“

P: „Bei Treppen, da fühle ich sowieso mehr mit den Beinen.“

U: „Haben Sie das Gefühl, dass sich räumlich etwas für Sie verändert hat?“

P: „Nein. Wenn ich auf einen Tisch schaue, das sagt mir nichts ... das ist irgendwie leerer Raum ... ich weiß dann nicht wie groß der ist, wie weit es von einer Seite zur anderen ist. Wenn was drauf steht, ist es irgendwie besser ... Leerer Raum ist für mich irgendwie nicht richtig da ..."

$\mathrm{U:} \mathrm{„Ist} \mathrm{Anstoßen} \mathrm{ein} \mathrm{Problem?“}$

P: „Ja, ja ... Ich glaube, ich bin zu wenig aufmerksam. Wenn ich mich auf eines konzentriere, kann ich schwer auf was daneben auch noch achten ... Und dann ist es ganz bestimmt so, dass ich die Entfernung einfach falsch einschätze ... ich glaub‘, in alle Richtungen ... “

U: „Im Bett zu liegen, ist das auch schwierig?“

P: „Ich bin noch nie rausgefallen, habe mich aber total verletzt. Ich wusste nicht, was quer und was längsseitig ist. Als ich mir vorstellen sollte, wo das Kopfende sein könnte, hatte ich immer das Gefühl, ich muss schauen, dass ich nicht herausfalle. Mittlerweile kann ich mich ganz gut im Bett orientieren.“

stimmten Bereichen des Raumes Folgebewegungen durchführen.

Mit dieser Technik gelingt es ohne großen apparativen Aufwand, den funktionalen Suchbereich (in dem der Patient Dinge beachtet) zumindest kurzfristig zu erweitern. Zudem kann manchmal auch die Fixation auf ein zu ergreifendes Objekt gelenkt werden. Verbale Kommandos sind meist nur bedingt erfolgreich. 
Tab.3 Darstellung und Erläuterung der einzelnen Teilaspekte des Balínt-Holmes-Syndroms.

\begin{tabular}{|c|c|}
\hline Störungsaspekt & Defizit \\
\hline Ätiologie & $\begin{array}{l}\text { bilaterale Posterior-Media-Grenzzoneninfarkte } \\
\text { Tumoren } \\
\text { zerebrale Hypoxie } \\
\text { Morbus Alzheimer }\end{array}$ \\
\hline Läsionslokalisation & $\begin{array}{l}\text { bilateral parieto-okzipital, selten bilateral frontal oder Stammganglien } \\
\text { diffus-disseminiert bei degenerativen Hirnerkrankungen (Morbus Alzheimer) }\end{array}$ \\
\hline Simultanwahrnehmung & $\begin{array}{l}\text { deutliche Einengung des visuellen Überblicks in beiden Halbfeldern bis hin zur Unfähigkeit, mehr als ein/ } \\
\text { wenige Objekte visuell simultan zu erfassen. }\end{array}$ \\
\hline optische Ataxie & $\begin{array}{l}\text { gestörte Auge-Hand-Koordination: } \\
\text { Vorbeizeigen und -greifen mit einer oder beiden Händen nach visuell präsentierten Objekten; besonders } \\
\text { ausgeprägt im kontralateralen Halbraum und in der Entfernung (nicht motorisch bedingt) } \\
\text { bei vorheriger Fixation des Objektes im zentralen Gesichtsfeldbereich gelingt das Greifen deutlich besser }\end{array}$ \\
\hline Fixation und Augenbewegungen & $\begin{array}{l}\text { spasmodische („klebende“) oder unruhige Fixation } \\
\text { visuelles Absuchen im Raum gravierend gestört }\end{array}$ \\
\hline $\begin{array}{l}\text { Raumwahrnehmung, räumliches } \\
\text { Gedächtnis }\end{array}$ & $\begin{array}{l}\text { gestörte Wahrnehmung von Entfernung, Distanz, Richtung und Position im Raum } \\
\text { gestörte längerfristige Speicherung solcher räumlichen Aspekte }\end{array}$ \\
\hline $\begin{array}{l}\text { Sehschärfe, Kontrastsehen, Gesichts- } \\
\text { feld, Stereosehen, Fusion }\end{array}$ & $\begin{array}{l}\text { Sehschärfe für Einzelzeichen meist intakt, für Reihenzeichen aufgrund des Simultansehens gestört } \\
\text { Kontrast- und Stereosehen sowie Fusion können intakt sein } \\
\text { assoziierte Gesichtsfeldausfälle sind häufig }\end{array}$ \\
\hline $\begin{array}{l}\text { Lesen, Schreiben, Zeichnen, visuelle } \\
\text { Orientierung, Selbsthilfe }\end{array}$ & $\begin{array}{l}\text { oft hochgradig beeinträchtigt } \\
\text { Lesen von Einzelworten relativ erhalten } \\
\text { massive Orientierungsstörung im Raum } \\
\text { Anziehen, Transfers und Mobilität infolge der anderen Defizite deutlich beeinträchtigt }\end{array}$ \\
\hline
\end{tabular}

\section{Greifen verbessern}

Durch die Verbesserung der Fixation normalisieren sich meist auch die Greifleistungen (optische Ataxie), da die Patienten während des Greifaktes den Zielgegenstand gar nicht oder ungenau fixiert haben. Lenkt der Therapeut die Aufmerksamkeit vor dem Greifen auf das Zielobjekt, verbessert sich meist die Greifleistung. Dies kann beispielsweise dadurch erreicht werden, dass man den Patienten ein spezifisches Objektmerkmal beschreiben lässt, das charakteristisch ist für das Objekt (Beispiel: Farbe der Kappe eines Stiftes) und nur bei genauer Fixation gesehen werden kann.

Das Ergreifen von Gegenständen, die vorher genau fixiert wurden, gelingt meist deutlich besser als das Greifen nach peripher gelegenen Gegenständen im linken oder rechten Halbraum.

\section{Simultansehen fördern}

Die Störung des Simultansehens äußert sich meist so, dass von mehreren Objekten (besonders wenn sie sich teilweise überlappen) nur eines oder wenige beachtet werden. Nach unseren Erfahrungen muss diese Störung ebenfalls spezifisch behandelt werden, da sie eine wichtige Voraussetzung für das Lesen ist (s.u.). Hierfür bieten sich am ehesten Alltagsobjekte an, die deutlich hinsichtlich ihrer Objektmerkmale auch für den Patienten unterscheidbar sind (Farbe, Größe,
Form der Objekte). Der Patient soll hierbei zunächst einen Gegenstand beschreiben, anschließend danach greifen und den Blick zum nächsten Gegenstand bewegen. Kommt es schon mit zwei Objekten zum Ausblenden eines Gegenstandes, so bietet es sich an, den Patienten die Augen schließen zu lassen oder ihm ein Blatt Papier kurz vor die Augen zu halten, und anschließend die Übung fortzusetzen. Die Anzahl der zu suchenden bzw. abzusuchenden Objekte sollte dann schrittweise gesteigert werden; anschließend können auch ähnlichere Objekte verwendet werden.

\section{Leseübungen}

Lesen erfordert zusätzliche okulomotorische Kontrollprozesse. Im ersten Behandlungsschritt sollten die Anzahl der Buchstaben pro Wort und die Wörter pro Seite deutlich reduziert werden. In Extremfällen kann manchmal nur in einer Zeile gearbeitet werden. Kurze, geläufige Worte sollten verwendet werden. Idealerweise sollte zunächst nur ein Wort dargeboten werden (um die Simultanagnosie zu umgehen). Spezifische Therapieprogramme enthalten ein Therapiemodul, welches die sequenzielle Darbietung einzelner Wörter an den jeweiligen Textpositionen erlaubt, um so den Patienten wieder an das Lesen einer Zeile, den Zeilensprung und schließlich eines ganzen Textes heranzuführen. 


\section{Scheinbewegungen vermeiden}

Viele Patienten mit Balínt-Holmes-Syndrom berichten zeitweilig über Scheinbewegungen von Objekten. Dies kann von der Schwierigkeit herrühren, zwischen eigenen Augenbewegungen und Bewegungen externer Objekte richtig zu unterscheiden. Praktisch äußert sich dies darin, dass das Orts- oder Positionsgedächtnis („Welches Objekt resp. welche Person war wo?") meist deutlich gestört ist.

Ein weiteres Behandlungsziel sollte deshalb die Verbesserung der Objekt-Positions-Relationen im zweidimensionalen und später dreidimensionalen Raum sein. Dies kann mit einfachen, farbig unterschiedlichen Alltagsgegenständen geübt werden (z.B. roter Textmarker links, grüner Anspitzer rechts). Die Farbe hilft dem Patienten in der Unterscheidung der beiden Objekte, da ihre räumliche Position oft nicht eindeutig erscheint. Klagen Patienten über störende Nachbilder oder Scheinbewegungen von Objekten, so hilft manchmal die Aufforderung, für ein paar Sekunden die Augen zu schließen.

\section{THERAPIE \\ Grundsätze in der Behandlung des Balínt- Holmes-Syndroms}

Bei der Behandlung des Balínt-Holmes-Syndroms ist es wichtig, nach erhaltenen Leistungen zu suchen, die zur Kompensation der Defizite eingesetzt werden können. Dies setzt eine individuelle Therapieplanung voraus. Mögliche Ansatzpunkte bieten eine Verbesserung der Fixation, des Greifens und des Simultansehens sowie Leseübungen und das Vermeiden von Scheinbewegungen. Ansonsten gilt es, die Therapie alltagsorientiert zu gestalten, sodass der Patient maximal von den Maßnahmen profitiert.

Wenngleich es noch keine evidenzbasierten Studien zur Therapie von Patienten mit Balínt-Holmes-Syndrom gibt, so können doch einige hilfreiche Tipps festgehalten werden (s. Infobox).

\section{PRAXIS}

Tipps

- Jeder dieser Patienten ist anders. Deshalb lohnt es sich, individuell nach erhaltenen Leistungen zu suchen, auf denen man in der Therapie aufbauen kann. Diese können bei verschiedenen Personen sehr unterschiedlich sein (z. B. anderer Sinneskanal; Orientierung am eigenen Körper manchmal besser; Altwissen; kognitive Leistungen, Humor etc.).

- Die Behandlung muss in den Alltag hinein ausgedehnt werden (oder gleich in diesem Setting stattfinden), sonst profitiert der Patient nicht davon.

- Der Grad der Kompensationsfähigkeit hängt auch von der relativen Intaktheit kognitiver Leistungen ab (Altgedächtnis, Arbeitsgedächtnis, Aufmerksamkeitsleistungen, Sprache, Exekutivfunktionen etc.).

- Wenngleich viele Störungen aufgrund der ausgedehnten Schädigungen meist unverändert auf der Testebene bestehen bleiben, können manche Patienten sehr wohl lernen, besser mit ihren Defiziten im Alltag umzugehen. Dies kann etwa durch die Verwendung anderer Sinneskanäle, anderer Lösungsstrategien, den Einsatz technischer Hilfen oder von Hilfspersonen gelingen.

\section{Zusammenfassung}

Raumorientierungsstörungen können sich auf vielfältige Weise manifestieren. Die wichtigsten zu dem Symptomkomplex gehörenden Störungen sind der Neglect, visuelle Raumorientierungsstörungen und das BalíntHolmes-Syndrom.

Zugrundeliegende Läsionen können vielfältig sein. Die posterior gelegenen Regionen des Okzipital-, Temporal- und Parietallappens spielen hierbei jedoch eine übergeordnete Rolle. Zudem können frontale sowie subkortikale Regionen an der Entstehung von Raumorientierungsstörungen beteiligt sein.

Als Neglect bezeichnet man das Vernachlässigen der kontraläsionalen Raumhälfte sowie den verminderten Gebrauch der kontraläsionalen Extremitäten in dem dazu korrespondierenden Teil des Raumes. Es werden verschiedene Formen des Störungsbildes unterschieden: Es existieren der raumbezogene und der objektbezogene Neglect sowie die Neglectdyslexie und der Body Neglect. Typische Symptome sind das Nicht-Reagieren auf sensorische Reize in der meist linken Raumhälfte, das Anstoßen an Hindernisse dort und verlängerte Suchzeiten beim Finden von Gegenständen oder beim Navigieren im Raum.

Die oftmals fehlende Krankheitseinsicht, die Unawareness oder Anosognosie der Patienten mit Neglect, erfordert eine detaillierte Selbst- und Fremdanamnese sowie eine Verhaltensbeobachtung. 
Visuelle Raumorientierungsstörungen werden in $4 \mathrm{Ka}$ tegorien eingeteilt: räumlich-perzeptive, räumlich-kognitive, räumlich-konstruktive und räumlich-topografische Störungen. Während räumlich-perzeptive Störungen eher basale visuelle Leistungen betreffen, beziehen sich die anderen 3 Subtypen auf höhere kognitive Leistungen. Das Störungsbild der visuellen Raumorientierungsstörungen ist gekennzeichnet durch eine fehlerhafte Eigenbewegung im Raum, eine gestörte Interaktion mit externen Objekten, fehlerhafte visuomotorische Kontrolle und eine verminderte Fähigkeit sich in vertrauten und insbesondere neuen Umgebungen zurechtzufinden.

Zum Balínt-Holmes-Syndrom gehören verschiedene Symptome wie die optische Ataxie, die Simultanagnosie sowie Raumverarbeitungs- und Blickbewegungsstörungen. Die Symptome treten entweder als Vollbild gemeinsam oder auch isoliert auf (letzteres meist nach unilateraler Läsion). Patienten berichten Probleme beim zielgerichteten Greifen sowie Schwierigkeiten beim Einschätzen von Positionen und Distanzen. Zudem zeigen die Betroffenen einen eingeschränkten visuellen Überblick.

Der Rehabilitationserfolg wird maßgeblich von der Behandlung der Raumorientierungsstörungen beeinflusst. Es gilt in jedem Fall, die für den Patienten relevanten Alltagsprobleme zu erfassen und erhaltene Teilfunktionen zu identifizieren und diese gezielt zu trainieren. Ansonsten existieren sowohl Bleistift- als auch computerbasierte Verfahren, um die einzelnen Symptome der Krankheitsbilder nachweislich zu verbessern.

\section{Interessenkonflikt}

Erklärung zu finanziellen Interessen

Forschungsförderung erhalten: nein; Honorar/geldwerten Vorteil für Referententätigkeit erhalten: nein; Bezahlter Berater/interner Schulungsreferent/Gehaltsempfänger: nein; Patent/Geschäftsanteile/Aktien (Autor/Partner, Ehepartner, Kinder) an Firma (Nicht-Sponsor der Veranstaltung): nein; Patent/Geschäftsanteile/Aktien (Autor/Partner, Ehepartner, Kinder) an Firma (Sponsor der Veranstaltung): nein.

Erklärung zu nichtfinanziellen Interessen

Die Autorinnen/Autoren geben an, dass kein Interessenkonflikt besteht.

\section{KERNAUSSAGEN}

- Raumorientierungsstörungen können sich auf vielfältige Weise manifestieren. Die wichtigsten zu dem Symptomkomplex gehörenden Störungen sind

- der Neglect,

- visuelle Raumorientierungsstörungen und

- das Balínt-Holmes-Syndrom.

- Zugrundeliegende Läsionen können vielfältig sein; die wichtigsten sind die posterior gelegenen Regionen des Okzipital-, Temporal- und Parietallappens. Auch frontale und subkortikale Regionen können beteiligt sein.

- Als Neglect bezeichnet man das Vernachlässigen der kontraläsionalen Raumhälfte sowie den verminderten Gebrauch der kontraläsionalen Extremitäten. Es werden verschiedene Formen unterschieden: der raumbezogene, der objektbezogene Neglect, die Neglectdyslexie und der Body Neglect; oftmals bei fehlender Krankheitseinsicht (Unawareness oder Anosognosie), was eine detaillierte Selbst- und Fremdanamnese sowie Verhaltensbeobachtung notwendig macht.

- Visuelle Raumorientierungsstörungen werden in 4 Kategorien eingeteilt: räumlich-perzeptive, räumlich-kognitive, räumlich-konstruktive und räumlich-topografische Störungen. Während räumlich-perzeptive Störungen eher basale visuelle Leistungen betreffen, beziehen sich die anderen 3 Subtypen auf höhere kognitive Leistungen.

- Mit dem Balínt-Holmes-Syndrom sind verschiedene Symptome assoziiert wie die optische Ataxie, die Simultanagnosie sowie Raumverarbeitungs- und Blickbewegungsstörungen, die als Vollbild gemeinsam oder isoliert auftreten (letzteres meist nach unilateraler Läsion).

- Der Rehabilitationserfolg wird maßgeblich von der Behandlung der Raumorientierungsstörungen beeinflusst. Die für den Patienten relevanten Alltagsprobleme sollten erfasst, erhaltene Teilfunktionen identifiziert und gezielt werden. Darüber hinaus existieren sowohl Bleistift- als auch computerbasierte Verfahren, um die einzelnen Symptome der Krankheitsbilder nachweislich zu verbessern. 


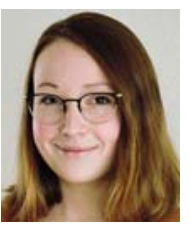

\section{Michelle Klein}

B.Sc. 2015-2020 Studium der Psychologie an der Universität des Saarlandes, Abschluss im Frühjahr 2020 mit Schwerpunkt Klinische Neuropsychologie. Wissenschaftliche Mitarbeiterin am Lehrstuhl für Klinische Neuropsychologie mit Schwerpunkten in der Lehre und Behandlung ambulanter neuropsychologischer Patienten. Außerdem Dozentin am Thein-Eifel-Institut in Andernach.

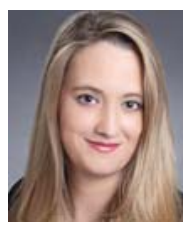

\section{Ann-Kathrin Bur}

M.Sc., tätig als wissenschaftliche Mitarbeiterin am Lehrstuhl für Klinische Neuropsychologie mit Schwerpunkten in der Lehre und Behandlung ambulanter neuropsychologischer Patienten und angestellt in den SHG Kliniken Sonnenberg als Psychologin M. Sc. bzw. Psychologische Psychotherapeutin in Ausbildung mit Schwerpunkt in der Behandlung von Patienten mit Suchtproblemen (und komorbiden Psychosen). Außerdem Dozentin am Rhein-Eifel-Institut in Andernach.

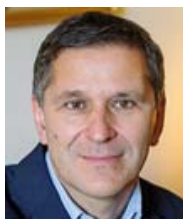

\section{Georg Kerkhoff}

Prof. Dr. phil., Klinischer Neuropsychologe, ist seit 2006 Professor für Klinische Neuropsychologie und Leiter der Neuropsychologischen UniAmbulanz an der Universität des Saarlandes (Saarbrücken). Seine Forschungsschwerpunkte sind die Klinik, Diagnostik und Therapie zerebral bedingter Seh- und Lesestörungen, multimodaler Neglect, Raumorientierungsstörungen sowie die evidenzbasierte Therapieforschung in den genannten Bereichen.

\section{Korrespondenzadresse}

\section{Prof. Dr. Georg Kerkhoff}

Klinische Neuropsychologie \&

Neuropsychologische Universitätsambulanz

Universität des Saarlandes

Gebäude A.1.3

Campus

66123 Saarbruecken

kerkhoff@mx.uni-saarland.de

Wissenschaftlich verantwortlich gemäß Zertifizierungsbestimmungen

Wissenschaftlich verantwortlich gemäß Zertifizierungsbestimmungen für diesen Beitrag ist Prof. Dr. med. HansChristoph Diener, Essen.

\section{Literatur}

[1] Kerkhoff G, Schmidt L. Neglect und assoziierte Störungen. 2. Überarbeitete Auflage. Göttingen: Hogrefe; 2018

[2] Karnath $\mathrm{HO}$, Rorden C. The anatomy of spatial neglect. Neuropsychologia 2012; 50: 1010-1017

[3] Sperber C, Karnath HO. Diagnostic validity of line bisection in the acute phase of stroke. Neuropsychologia 2016; 82: 200-204
[4] Kerkhoff G, Groh-Bordin C. Höhere visuelle Funktionen: Neglect, Raumorientierung, Balínt-Holmes-Syndrom und visuelle Agnosien. In: Neurorehabilitation. Berlin/Heidelberg: Springer; 2010: 207-222

[5] Utz KS, Keller I, Artinger F et al. Multimodal and multispatial deficits of verticality perception in hemispatial neglect. Neuroscience 2011; 188: 68-79

[6] Baier B, Suchan J, Karnath HO et al. Neural correlates of disturbed perception of verticality. Neurology 2012; 78: 728735

[7] Gillebert CR, Mantini D, Thijs V et al. Lesion evidence for the critical role of the intraparietal sulcus in spatial attention. Brain 2011; 134: 1694-1709

[8] Sack AT, Sperling JM, Prvulovic D et al. Tracking the mind's image in the brain II: transcranial magnetic stimulation reveals parietal asymmetry in visuospatial imagery. Neuron 2002; 35: 195-204

[9] Kerkhoff G, Marquardt C. VS-WIN - Computational analysis of visuospatial perception and cognition. München: MedCom; 2004

[10] Mack JL, Levine RN. The basis of visual constructional disability in patients with unilateral cerebral lesions. Cortex 1981; 17: 515-531

[11] Russell C, Deidda C, Malhotra P et al. A deficit of spatial remapping in constructional apraxia after right-hemisphere stroke. Brain 2010; 133: 1239-1251

[12] De Renzi E. Disorders of space exploration and cognition. New York: John Wiley \& Sons; 1982

[13] Epstein RA. Parahippocampal and retrosplenial contributions to human spatial navigation. Trends in cognitive sciences 12: 388-396

[14] Benton AL, Hamsher KD, Varney NR et al. Judgment of line orientation. New York: Oxford University Press; 1983

[15] Warrington EK, James M. Testbatterie Für Visuelle Objektund Raumwahrnehmung: Handanweisung; VOSP. Thames Vallery Test Company. 1992

[16] Riddoch M], Humphreys GW. Birmingham object recognition battery. Lawrence Erlbaum Associates; 1993

[17] Funk J, Finke K, Reinhart S et al. Effects of feedback-based visual line-orientation discrimination training for visuospatial disorders after stroke. Neurorehabil Neural Repair 2013; 27: $142-152$

[18] Kerkhoff G. Modulation and rehabilitation of spatial neglect by sensory stimulation. In: Progress in brain research.142 New York: Elsevier; 2003: 257-271

[19] Weinberg J, Piasetsky E, Diller L et al. Treating perceptual organization deficits in nonneglecting RBD stroke patients. J Clin Exp Neuropsychol 1982; 4: 59-75

[20] Kerkhoff G, Heldmann B. Balínt-Syndrom und assoziierte Störungen Anamnese - Diagnostik - Behandlungsansätze. Nervenarzt 1999; 70: 10859-869

\section{Bibliografie}

DOI https://doi.org/10.1055/a-0945-2011

Neurologie up2date 2020; 03: 179-193

(c) Georg Thieme Verlag KG Stuttgart · New York ISSN 2511-3453 


\section{Punkte sammeln auf CME.thieme.de}

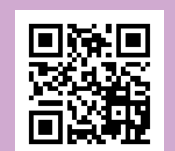

Diese Fortbildungseinheit ist in der Regel 12 Monate online für die Teilnahme verfügbar. Den genauen Einsendeschluss finden Sie unter https://cme.thieme.de.

Sollten Sie Fragen zur Online-Teilnahme haben, finden Sie unter https://cme.thieme.de/hilfe eine ausführliche Anleitung. Wir wünschen viel Erfolg beim Beantworten der Fragen!

Unter https://eref.thieme.de/CXDCIIF oder über den QR-Code kommen Sie direkt zur Startseite des Wissenstests.

VNR 2760512020158724613

\section{Frage 1}

Eine der folgenden Aussagen trifft bezüglich Neglect-Patienten nicht zu. Welche?

A Patienten haben Schwierigkeiten, zwei Objekte gleichzeitig wahrzunehmen.

B Meistens liegt eine rechtshemisphärische temporo-parietale Schädigung zugrunde.

C Oftmals fehlt den Patienten die Einsicht für ihre Defizite. Dieses Phänomen bezeichnet man als Unawareness.

D Man unterscheidet zwischen raum- und objektbezogenem Neglect.

E Patienten kollidieren oft mit Hindernissen und reagieren vermindert auf Reize in der kontraläsionalen Raumhälfte.

\section{Frage 2}

Welches ist kein anerkanntes Therapieverfahren bei der Behandlung eines Neglects?
A Neuromodulation
B visuelles Explorationstraining
C visuomotorische Prismenadaptation
D Zeichenaufgaben
E Aufmerksamkeitstraining

\section{Frage 3}

Der Patient zeigt Probleme, sich sowohl in neuen als auch vertrauten Umgebungen zurechtzufinden. Ursächlich können welche Störungsbilder sein?
A Balínt-Holmes-Syndrom und Neglect
B räumlich-topografische und räumlich-kognitive Störungen
C räumlich-topografische Störungen und Neglect
D räumlich-konstruktive und räumlich-perzeptive Störungen
E räumlich-kognitive Störungen und Neglect

\section{Frage 4}

Die optische Ataxie ist eins der Symptome des Balínt-HolmesSyndroms. Was versteht man darunter?

A die Unfähigkeit, mehrere Dinge gleichzeitig wahrzunehmen

B die Probleme, mentale Rotationen durchzuführen

C die fehlende Einsicht bezüglich eigener Defizite

D unruhige Fixationen beider Augen

E Probleme beim zielgerichteten Greifen

\section{Frage 5}

Welcher Ansatz könnte bei der Behandlung des Balínt-HolmesSyndroms (BHS) Erfolg bringen?
A visuelles Feedback-Training
B Entwicklung individueller Strategien, um entstandene Defizite zu kompensieren
C räumlich-konstruktives Training
D massiertes Üben (Restitution)
E Training nicht alltagsrelevanter, übergeordneter Funk- tionen

\section{Frage 6}

Eines der folgenden Verfahren ist kein Subtest aus der Visual Object and Space Perception Battery (VOSP) von Warrington u. James. Welches?
A Positionsvergleich
B Punktezählen
C Linienhalbierung
D Würfelzählen
E Zahlenlokalisieren 


\section{Punkte sammeln auf CME.thieme.de}

Fortsetzung ...

\section{Frage 7}

Was versteht man unter räumlich-topografischen Störungen?

A Navigationsdefizite im vorgestellten oder realen dreidimensionalen Raum

B das Nichtbeachten von Reizen auf der kontraläsionalen Seite

C Abweichungen von der tatsächlichen Vertikalen bzw. Horizontalen bei der Beurteilung, wann eine Linie, die schrittweise rotiert wird, vertikal bzw. horizontal ausgerichtet ist

D die Unfähigkeit, einzelne Elemente einer Figur mit der Hand zu einem Ganzen zusammenzusetzen

E Einbußen visueller Raumoperationen, die über die Wahrnehmungsleistung hinaus, oder ohne eine solche, eine mentale Raumoperation erfordern

\section{Frage 8}

Wie bezeichnet man die fehlende Wahrnehmung der eigenen Krankheit durch die Betroffenen?
A Anosognosie
B Asomatognosie
C Aphasie
D Apraxie
E Agnosie

\section{Frage 9}

Ein Patient berichtet nach einem bilateralen posterioren Schlaganfall Probleme im Alltag, beispielsweise beim Lesen oder Ankleiden. Er verfehle oft eine Treppenstufe oder auch Dinge, nach denen er versuche zu greifen. Er sei vorher leidenschaftlicher Tennisspieler gewesen, doch nun habe er die Lust daran verloren, da er immer wieder am Ball vorbeischlage oder die Entfernung zum Netz falsch einschätze. Der Befund des Augenarztes ist allerdings negativ. Auf welche Störung hin untersuchen Sie den Mann?
A räumlich-topografische Störung
B Hemianopsie
C Neglect
D Balínt-Holmes-Syndrom
E Reduktion der Sehstärke

\section{Frage 10}

Eines der genannten Einzelsymptome gehört nicht zur Gruppe der räumlich-perzeptiven Störungen. Welches?
A Defizite in der Orientierungsschätzung
B Defizite in der Längeneinschätzung
C Defizite bei simultaner Wahrnehmung mehrerer Gegen- stände
D Defizite in der Positionswahrnehmung
E Defizite in der subjektiven Geradeausrichtung 